\title{
ON A STUDY OF BINOMIAL FORM TO THE NEW $(S, T)$-JACOBSTHAL SEQUENCE
}

\author{
A. A. Wani, S. Halici, T. A. Tarray
}

Abstract. Many $(s, t)$-type of sequences has been introduced earlier such as $(s, t)$-Fibonacci sequence, $(s, t)$-Lucas sequence, $(s, t)$-Jacobsthal sequence, $(s, t)$ Jacobsthal-Lucas sequence etc. However in this article, we give a new type of $(s, t)$-Jacobsthal sequence $\left\langle U_{n}(s, t)\right\rangle_{n \in \mathbb{N}}$

$$
U_{n}=i U_{n-1}+2 U_{n-2}, n \geq 2 \text { and } U_{0}=s-2 t, U_{1}=i(s-t)
$$

where $i=\sqrt{-1}$ and $s, t \in \mathbb{Z}^{+}$. Next we define a binomial form $\left\langle X_{n}(s, t)\right\rangle_{n \in \mathbb{N}}$ to the new $(s, t)$-Jacobsthal sequence and then some fundamental properties for the binomial form $\left\langle X_{n}(s, t)\right\rangle_{n \in \mathbb{N}}$ are obtained. Furthermore a new kind of matrix sequence $\left\langle Z_{n}(s, t)\right\rangle_{n \in \mathbb{N}}$ will be presented for the binomial form $\left\langle X_{n}(s, t)\right\rangle_{n \in \mathbb{N}}$.

2010 Mathematics Subject Classification: 11B37, 11B39.

Keywords: Jacobsthal Sequences, $(s, t)$-type Sequences, Binomial Form and Matrix Sequence.

\section{INTRODUCTION}

In the mathematical world, the Fibonacci sequence (see [1]) have great importance and plays an important role almost in the every arena of science. Some sequences such as Jacobsthal and Jacobsthal-Lucas sequences etc have similar structure to the Fibonacci sequence and in another words, we can say that these sequences are the extensions or generalizations of Fibonacci sequence.

In 1961 Horadam [2] introduced the first ever generalization of Fibonacci sequence and denoted it by $\left\langle H_{n}\right\rangle$.

$$
H_{n}=H_{n-1}+H_{n-2}, \quad n \geq 3 \text { and } H_{0}=p, H_{1}=p+q
$$


A. A. Wani, S. Halici, T. A. Tarray - On a Study of Binomial Form ...

where $p$ and $q$ are arbitrary integers. Then in 1965 Horadam introduced another generalized Fibonacci sequence $\left\langle W_{n}\right\rangle$ called as Horadam sequence (see [3]). The sequence $\left\langle W_{n}\right\rangle$ is generated by the following recurrence relation

$$
W_{n}=p W_{n-1}-q W_{n-2}, \quad n \geq 2 \text { and } W_{0}=a, W_{1}=b
$$

where $a, b, p$ and $q$ are real constants. After that a lot of work has been done to study the generalizations of Fibonacci sequences by several methods.

In formal terms, a complex sequence is a function whose domain is the positive integers and co-domain is a set of the complex numbers. Gaussian numbers were first investigated in 1832 by the German mathematician Karl Friedrich Gauss. A Gaussian number is a complex number $Z=a+i b$, where $a$ and $b$ are any integers and $i=\sqrt{-1}$. In 1963 Horadam [4] considered the generalized Fibonacci sequence (1.1) and then delineated generalized complex Fibonacci sequence $\left\langle D_{n}\right\rangle$.

$$
\begin{aligned}
D_{n} & =H_{n}+i H_{n+1}, \quad n \geq 1 \\
& =\left(p_{H}-q_{H}+i q_{H}\right) F_{n}+\left(q_{H}+i p_{H}\right) F_{n+1}, \quad n \geq 1 \\
& =D_{n-1}+D_{n-2}, \quad n \geq 3
\end{aligned}
$$

with $D_{1}=(1+i) p_{H}+q_{H}$ and $D_{2}=p_{H}+q_{H}+i\left(2 p_{H}+q_{H}\right)$.

As a special case of equation (1.3) the author defined a complex Fibonacci sequence $\left\langle C_{n}\right\rangle$ such that

$$
\begin{aligned}
C_{n} & =F_{n}+i F_{n+1}, \quad n \geq 1 \\
& =C_{n-1}+C_{n-2}, \quad n \geq 3
\end{aligned}
$$

with $C_{1}=1+i$ and $C_{2}=1+2 i$.

Jordan [5] in 1965 presented a Gaussian Fibonacci sequence $\left\langle G F_{n}\right\rangle$ and established some results between Gaussian Fibonacci sequence and classical Fibonacci sequence.

$$
G F_{n}=G F_{n-1}+G F_{n-2}, \quad n \geq 2 \text { and } G F_{0}=i, G F_{1}=1
$$

Later on Berzsenyi [6], Harman [7] and Pethe [8] used different approaches of extensions of Fibonacci numbers on the complex plane.

Now we discuss some other literature where the authors studied the generalizations of Jacobsthal numbers and Jacobsthal-Lucas numbers. Asci ans Gurel [9] introduced and studied Gaussian Jacobsthal $\left\langle G J_{n}\right\rangle$ and Gaussian Jacobsthal-Lucas $\left\langle G j_{n}\right\rangle$ numbers.

$$
G J_{n+1}=G J_{n}+2 G J_{n-1}, \quad n \geq 1 \text { and } G J_{0}=\frac{i}{2}, G J_{1}=1
$$




$$
G j_{n+1}=G j_{n}+2 G j_{n-1}, \quad n \geq 1 \text { and } G j_{0}=2-\frac{i}{2}, G j_{1}=1+2 i .
$$

Asci ans Gurel [10] defined the polynomials of Gaussian Jacobsthal and Gaussian Jacobsthal-Lucas numbers. Catarino et al. [11] studied the new generalizations of Jacobsthal and Jacobsthal-Lucas sequences. Uygun [12] also defined new generalizations for Jacobsthal and Jacobsthal-Lucas sequences called $p(x)$-Jacobsthal polynomial sequences $\left\langle J_{p, n}(x)\right\rangle$ and $p(x)$-Jacobsthal-Lucas polynomial sequences $\left\langle C_{p, n}(x)\right\rangle$ by

$$
\begin{aligned}
& J_{p, n}(x)=p(x) J_{p, n-1}(x)+2 J_{p, n-2}(x), n \geq 2 \text { and } J_{p, 0}(x)=0, J_{p, 1}(x)=1 \\
& C_{p, n}(x)=p(x) C_{p, n-1}(x)+2 C_{p, n-2}(x), n \geq 2 \text { and } C_{p, 0}(x)=2, C_{p, 1}(x)=p(x)
\end{aligned}
$$

where $p(x)$ is a polynomial with real coefficients.

In [13] a sequence $\left\langle b_{n}\right\rangle_{n \in \mathbb{Z}_{0}}$ is the binomial transform to the sequence $\left\langle a_{n}\right\rangle_{n \in \mathbb{Z}_{0}}$ if

$$
b_{n}=\sum_{k=0}^{n}\left(\begin{array}{l}
n \\
k
\end{array}\right) a_{k}
$$

Chen [13] obtained various identities related to binomial transform. In [14] and [15] the authors discussed the binomial transforms to the Dold and Fibonacci-Like sequences respectively.

From past several years many authors investigated the generalizations of Fibonacci, Lucas, Jacobsthal sequences etc by adding parameters $s$ and $t$ to the recurrence relations of these sequences then named the resulted sequences as $(s, t)$-type sequences. In addition to this they also defined the matrix sequences for $(s, t)$-type sequences and called the matrix sequences as $(s, t)$-type matrix sequences. A matrix sequence is the sequence in which the terms of the sequences are in the form of matrices and the elements of these matrices are the terms of general sequences. In 2008 Civciv and and Turkmen [16] presented $(s, t)$-Fibonacci sequence $\left\langle F_{n}(s, t)\right\rangle$ and $(s, t)$-Fibonacci matrix sequence $\left\langle\mathcal{F}_{n}(s, t)\right\rangle$.

$$
\begin{gathered}
F_{n+1}(s, t)=s F_{n}(s, t)+t F_{n-1}(s, t), n \geq 1 \text { and } F_{0}(s, t)=0, F_{1}(s, t)=1 \\
\mathcal{F}_{n+1}(s, t)=s \mathcal{F}_{n}(s, t)+t \mathcal{F}_{n-1}(s, t), \quad n \geq 1
\end{gathered}
$$

with $\mathcal{F}_{0}(s, t)=\left[\begin{array}{ll}1 & 0 \\ 0 & 1\end{array}\right], \mathcal{F}_{1}(s, t)=\left[\begin{array}{cc}s & 1 \\ t & 0\end{array}\right]$

whereas $\mathcal{F}_{n}(s, t)=\left[\begin{array}{cc}F_{n+1}(s, t) & F_{n}(s, t) \\ t F_{n}(s, t) & t F_{n-1}(s, t)\end{array}\right]$ for $s>0, t \neq 0, s^{2}+4 t>0$. 
A. A. Wani, S. Halici, T. A. Tarray - On a Study of Binomial Form ...

In 2011 Yazlik et al. [17] introduced the generalizations of $(s, t)$-Fibonacci sequence and $(s, t)$-Fibonacci matrix sequence by defining the sequences $\left\langle G_{n}(s, t)\right\rangle_{n \in \mathbb{N}}$ called the generalized $(s, t)$-Fibonacci sequence and $\left\langle\mathfrak{R}_{n}(s, t)\right\rangle_{n \in \mathbb{N}}$ called the generalized $(s, t)$-Fibonacci matrix sequence. The sequences $\left\langle G_{n}(s, t)\right\rangle_{n \in \mathbb{N}}$ and $\left\langle\mathfrak{R}_{n}(s, t)\right\rangle_{n \in \mathbb{N}}$ are recurrently defined by

$$
G_{n+1}(s, t)=s G_{n}(s, t)+t G_{n-1}(s, t), n \geq 1 \text { and } G_{0}(s, t)=a, G_{1}(s, t)=b s
$$

and

$$
\mathfrak{R}_{n+1}(s, t)=s \mathfrak{R}_{n}(s, t)+t \mathfrak{R}_{n-1}(s, t), \text { for } n \geq 1
$$

with $\mathfrak{R}_{0}(s, t)=\left[\begin{array}{cc}b s & a \\ a t & (b-a) s\end{array}\right], \mathfrak{R}_{1}(s, t)=\left[\begin{array}{cc}b s^{2}+a t & b s \\ b s t & a t\end{array}\right]$ whereas $\mathfrak{R}_{n}(s, t)=$ $\left[\begin{array}{cc}G_{n+1}(s, t) & G_{n}(s, t) \\ t G_{n}(s, t) & t G_{n-1}(s, t)\end{array}\right]$ for $s>0, t \neq 0, s^{2}+4 t>0$ and $a, b \in \mathbb{R}$.

Again in 2015 Ipek et al. [18] delineated the another generalized $(s, t)$-Fibonacci sequence $\left\langle G_{n}(s, t)\right\rangle_{n \in \mathbb{N}}$ and its matrix sequence $\left\langle\Re_{n}(s, t)\right\rangle_{n \in \mathbb{N}}$ by

$$
G_{n+1}(s, t)=s G_{n}(s, t)+t G_{n-1}(s, t), n \geq 1 \text { and } G_{0}(s, t)=a_{0}, G_{1}(s, t)=a_{1}
$$

and

$$
\mathfrak{R}_{n+1}(s, t)=s \mathfrak{R}_{n}(s, t)+t \mathfrak{R}_{n-1}(s, t), \quad n \geq 1
$$

with $\mathfrak{R}_{0}(s, t)=\left[\begin{array}{cc}a_{1} & a_{0} \\ t a_{0} & a_{1}-s a_{0}\end{array}\right], \mathfrak{R}_{1}(s, t)=\left[\begin{array}{cc}s a_{1}+t a_{0} & a_{1} \\ t a_{1} & t a_{0}\end{array}\right]$ whereas $\mathfrak{R}_{n}(s, t)=$ $\left[\begin{array}{cc}G n+1(s, t) & G_{n}(s, t) \\ t G_{n}(s, t) & t G_{n-1}(s, t)\end{array}\right]$ for $s>0, t \neq 0, s^{2}+4 t>0$ and $a_{0}, a_{1} \in \mathbb{R}$.

Yazlik et al. [19] applied binomial transforms to the $(s, t)$-Fibonacci matrix sequence $(1.12)$ and generalized $(s, t)$-Fibonacci matrix sequence (1.14). Uygun [20] presented $(s, t)$-Jacobsthal sequence $\left\langle\hat{\jmath}_{n}(s, t)\right\rangle$ and $(s, t)$-Jacobsthal-Lucas sequence $\left\langle\hat{c}_{n}(s, t)\right\rangle$ such that

$$
\begin{aligned}
& \hat{\jmath}_{n}(s, t)=s \hat{\jmath}_{n-1}(s, t)+2 t \hat{\jmath}_{n-2}(s, t), \quad n \geq 2 \text { and } \hat{\jmath}_{0}(s, t)=0, \hat{\jmath}_{1}(s, t)=1 \\
& \hat{c}_{n}(s, t)=s \hat{c}_{n-1}(s, t)+2 t \hat{c}_{n-2}(s, t), \quad n \geq 2 \text { and } \hat{c}_{0}(s, t)=2, \hat{c}_{1}(s, t)=s
\end{aligned}
$$


where $s>0, t \neq 0$ and $s^{2}+8 t>0$.

Then in [21] the authors defined $(s, t)$-Jacobsthal matrix sequence $\left\langle J_{n}(s, t)\right\rangle_{n \in \mathbb{N}}$ and $(s, t)$-Jacobsthal-Lucas matrix sequence $\left\langle C_{n}(s, t)\right\rangle_{n \in \mathbb{N}}$ as

$$
J_{n+1}(s, t)=s J_{n}(s, t)+2 t J_{n-1}(s, t), \quad n \geq 1
$$

with $J_{0}(s, t)=\left[\begin{array}{ll}1 & 0 \\ 0 & 1\end{array}\right], J_{1}(s, t)=\left[\begin{array}{ll}s & 2 \\ t & 0\end{array}\right]$ whereas $J_{n}(s, t)=\left[\begin{array}{cc}\hat{\jmath}_{n+1}(s, t) & \hat{\jmath}_{n}(s, t) \\ t \hat{\jmath}_{n}(s, t) & t \hat{\jmath}_{n-1}(s, t)\end{array}\right]$ and

$$
C_{n+1}(s, t)=s C_{n}(s, t)+2 t C_{n-1}(s, t), \quad n \geq 1
$$

with $C_{0}(s, t)=\left[\begin{array}{cc}s & 4 \\ 2 t & -s\end{array}\right], C_{1}(s, t)=\left[\begin{array}{cc}s^{2}+4 t & 2 s \\ s t & 4 t\end{array}\right]$

whereas and $C_{n}(s, t)=\left[\begin{array}{cc}\hat{c}(s, t) & \hat{c}_{n}(s, t) \\ t \hat{c}_{n}(s, t) & t \hat{c}_{n-1}(s, t)\end{array}\right]$ for $t \neq 0, s^{2}+8 t \neq 0$.

Uygun [22] gave some summation identities for the $(s, t)$-Jacobsthal and $(s, t)$ Jacobsthal-Lucas matrix sequences.

In 2016 Uygun and Uslu [23] studied the generalizations of $(s, t)$-Jacobsthal and $(s, t)$-Jacobsthla-Lucas sequences as well as generalizations of their matrix ones. Thus in [23] the authors presented $(s, t)$-generalized Jacobsthal sequence $\left\langle G_{n}(s, t)\right\rangle_{n \in \mathbb{N}}$ and $(s, t)$-generalized Jacobsthal matrix sequence $\left\langle\mathfrak{R}_{n}(s, t)\right\rangle_{n \in \mathbb{N}}$ by the following equations

$$
G_{n+1}(s, t)=s G_{n}(s, t)+2 t G_{n-1}(s, t), n \geq 1 \text { and } G_{0}(s, t)=a, G_{1}(s, t)=b s
$$

and

$$
\mathfrak{R}_{n+1}(s, t)=s \mathfrak{R}_{n}(s, t)+2 t \mathfrak{R}_{n-1}(s, t), \quad n \geq 1
$$

with $\mathfrak{R}_{0}(s, t)=\left[\begin{array}{cc}b s & 2 a \\ a t & (b-a) s\end{array}\right], \mathfrak{R}_{1}(s, t)=\left[\begin{array}{cc}b s^{2}+2 a t & 2 b s \\ b s t & 2 a t\end{array}\right]$ whereas $\mathfrak{R}_{n}(s, t)=$ $\left[\begin{array}{cc}G_{n+1}(s, t) & G_{n}(s, t) \\ t G_{n}(s, t) & t G_{n-1}(s, t)\end{array}\right]$ for $s>0, t \neq 0, s^{2}+4 t>0$ and $a, b \in \mathbb{R}$.

In the rest of the paper, we use symbols $\left\langle U_{n}\right\rangle,\left\langle X_{n}\right\rangle$ and $\left\langle Z_{n}\right\rangle$ instead of $\left\langle U_{n}(s, t)\right\rangle$, $\left\langle X_{n}(s, t)\right\rangle$ and $\left\langle Z_{n}(s, t)\right\rangle$. 
A. A. Wani, S. Halici, T. A. Tarray - On a Study of Binomial Form ...

\section{2. $(s, t)-J$ ACOBSTHAL SEQUENCE}

Definition 1. For $s, t \in \mathbb{Z}^{+}$and $i(=\sqrt{-1})$, the $(s, t)$-Jacobsthal sequence $\left\langle U_{n}\right\rangle_{n \in \mathbb{N}}$ is recurrently defined by

$$
U_{n}=i U_{n-1}+2 U_{n-2}, \quad n \geq 2
$$

with seeds $U_{0}=s-2 t$ and $U_{1}=i(s-t)$

The recurrence relation (2.1) have the characteristic equation $u^{2}-i u-2=0$ and suppose that $\theta$ and $\vartheta$ are the roots of this characteristic equation.

$$
\theta=\frac{\sqrt{7}+i}{2} \text { and } \vartheta=\frac{-(\sqrt{7}-i)}{2}
$$

\section{Binomial Form to the $(s, t)$-Jacobsthal Sequence}

In this section first and foremost we give a binomial form $\left\langle X_{n}\right\rangle$ of $(s, t)$ - Jacobsthal sequence $\left\langle U_{n}\right\rangle$ and after that a recurrence relation and Binet's formula for $\left\langle X_{n}\right\rangle$ are presented.

Definition 2. For $n \in \mathbb{Z}_{0}$, the binomial form to the $(s, t)$-Jacobsthal sequence $\left\langle U_{n}\right\rangle$ is defined by

$$
X_{n}=\sum_{l=0}^{n}\left(\begin{array}{l}
n \\
l
\end{array}\right) U_{l}
$$

Lemma 1. For $n \in \mathbb{Z}_{0}$, the following property holds for $\left\langle X_{n}\right\rangle$

$$
X_{n+1}=\sum_{l=0}^{n}\left(\begin{array}{l}
n \\
l
\end{array}\right)\left(U_{l}+U_{l+1}\right)
$$

Proof. Its proof can be easily obtained by using the relation $\left(\begin{array}{c}n+1 \\ l\end{array}\right)=\left(\begin{array}{l}n \\ l\end{array}\right)+$ $\left(\begin{array}{c}n \\ l-1\end{array}\right)$

Theorem 2. ( Recurrence relation for $\left\langle X_{n}\right\rangle$ ) If $s, t \in \mathbb{Z}^{+}$and $i(=\sqrt{-1})$, the recurrence relation of the binomial form $\left\langle X_{n}\right\rangle$ is given by

$$
X_{n+1}=(2+i) X_{n}+(1-i) X_{n-1}, \quad n \geq 1
$$

with $X_{0}=s-2 t \quad$ and $X_{1}=s(1+i)-t(2+i)$ 
Proof. Since

$$
\begin{aligned}
X_{n+1}= & \sum_{l=0}^{n}\left(\begin{array}{l}
n \\
l
\end{array}\right)\left(U_{l}+U_{l+1}\right) \\
& =U_{0}+U_{1}+\sum_{l=1}^{n}\left(\begin{array}{l}
n \\
l
\end{array}\right)\left(U_{l}+U_{l+1}\right) \\
& =U_{0}+U_{1}+\sum_{l=1}^{n}\left(\begin{array}{l}
n \\
l
\end{array}\right)\left(U_{l}+i U_{l}+2 U_{l-1}\right) \quad \text { By the Eqn. }(2.1) \\
& =U_{0}+U_{1}+\sum_{l=1}^{n}\left(\begin{array}{l}
n \\
l
\end{array}\right)\left[(1+i) U_{l}+2 U_{l-1}\right] \\
& =(1+i) \sum_{l=1}^{n}\left(\begin{array}{l}
n \\
l
\end{array}\right) U_{l}+2 \sum_{l=1}^{n}\left(\begin{array}{l}
n \\
l
\end{array}\right) U_{l-1}+U_{0}+U_{1} \\
= & (1+i) \sum_{l=1}^{n}\left(\begin{array}{l}
n \\
l
\end{array}\right) U_{l}+(1+i) U_{0}+2 \sum_{l=1}^{n}\left(\begin{array}{l}
n \\
l
\end{array}\right) U_{l-1}-(1+i) U_{0}+U_{0} \\
& \quad+U_{1} \\
= & (1+i) \sum_{l=0}^{n}\left(\begin{array}{l}
n \\
l
\end{array}\right) U_{l}+2 \sum_{l=1}^{n}\left(\begin{array}{l}
n \\
l
\end{array}\right) U_{l-1}-i U_{0}+U_{1} \\
= & (1+i) X_{n}+2 \sum_{l=1}^{n}\left(\begin{array}{l}
n \\
l
\end{array}\right) U_{l-1}-i U_{0}+U_{1} \quad \text { By the Eqn. }(3.1)
\end{aligned}
$$

By replacing $n$ by $n-1$, we get

$$
\begin{aligned}
X_{n}= & (1+i) X_{n-1}+2 \sum_{l=1}^{n-1}\left(\begin{array}{c}
n-1 \\
l
\end{array}\right) U_{l-1}-i U_{0}+U_{1} \\
= & i X_{n-1}+\sum_{l=0}^{n-1}\left(\begin{array}{c}
n-1 \\
l
\end{array}\right) U_{l}+2 \sum_{l=1}^{n-1}\left(\begin{array}{c}
n-1 \\
l
\end{array}\right) U_{l-1}-i U_{0}+U_{1} \\
= & i X_{n-1}+\sum_{l=1}^{n}\left(\begin{array}{c}
n-1 \\
l-1
\end{array}\right) U_{l-1}+2\left[\left(\begin{array}{c}
n-1 \\
1
\end{array}\right) U_{0}+\left(\begin{array}{c}
n-1 \\
2
\end{array}\right) U_{1}+\left(\begin{array}{c}
n-1 \\
3
\end{array}\right)\right. \\
& \left.U_{2}+\cdots+\left(\begin{array}{c}
n-1 \\
n-1
\end{array}\right) U_{n-2}+\left(\begin{array}{c}
n-1 \\
n
\end{array}\right) U_{n-1}\right]-i U_{0}+U_{1}
\end{aligned}
$$


After using the fact $\left(\begin{array}{c}n-1 \\ n\end{array}\right)=0$, we have

$$
\begin{aligned}
X_{n} & =i X_{n-1}+\sum_{l=1}^{n}\left(\begin{array}{c}
n-1 \\
l-1
\end{array}\right) U_{l-1}+2 \sum_{l=1}^{n}\left(\begin{array}{c}
n-1 \\
l
\end{array}\right) U_{l-1}-i U_{0}+U_{1} \\
X_{n} & =i X_{n-1}+\sum_{l=1}^{n}\left[\left(\begin{array}{c}
n-1 \\
l-1
\end{array}\right)+2\left(\begin{array}{c}
n-1 \\
l
\end{array}\right)\right] U_{l-1}-i U_{0}+U_{1} \\
& =i X_{n-1}+\sum_{l=1}^{n}\left[\left(\begin{array}{c}
n-1 \\
l-1
\end{array}\right)+2\left(\begin{array}{c}
n-1 \\
l
\end{array}\right)+2\left(\begin{array}{c}
n-1 \\
l-1
\end{array}\right)-2\left(\begin{array}{c}
n-1 \\
l-1
\end{array}\right)\right] U_{l-1} \\
& -i U_{0}+U_{1} \\
& =i X_{n-1}+\sum_{l=1}^{n}\left[\left(\begin{array}{l}
1-2 \\
l=1
\end{array}\right)\left(\begin{array}{c}
n-1 \\
l-1
\end{array}\right)+2\left(\begin{array}{l}
n \\
l
\end{array}\right)\right] U_{l-1}-i U_{0}+U_{1} \\
& =i X_{n-1}-\sum_{l=1}^{n}\left(\begin{array}{c}
n-1 \\
l-1
\end{array}\right) U_{l-1}+2 \sum_{l=1}^{n}\left(\begin{array}{l}
n \\
l
\end{array}\right) U_{l-1}-i U_{0}+U_{1} \\
& =i X_{n-1}-\sum_{l=0}^{n-1}\left(\begin{array}{c}
n-1 \\
l
\end{array}\right) U_{l}+2 \sum_{l=1}^{n}\left(\begin{array}{c}
n \\
l
\end{array}\right) U_{l-1}-i U_{0}+U_{1} \\
& =i X_{n-1}-X_{n-1}+2 \sum_{l=1}^{n}\left(\begin{array}{c}
n \\
l
\end{array}\right) U_{l-1}-i U_{0}+U_{1} \\
& =(i-1) X_{n-1}+2 \sum_{l=1}^{n}\left(\begin{array}{l}
n \\
l
\end{array}\right) U_{l-1}-i U_{0}+U_{1}
\end{aligned}
$$

Thus

$$
X_{n}-(i-1) X_{n-1}=2 \sum_{l=1}^{n}\left(\begin{array}{l}
n \\
l
\end{array}\right) U_{l-1}-i U_{0}+U_{1}
$$

Hence from the equation (3.4), we get

$$
\begin{aligned}
X_{n+1} & =(1+i) X_{n}+X_{n}-(i-1) X_{n-1} \\
& =(2+i) X_{n}+(1-i) X_{n-1}
\end{aligned}
$$

as required.

One can obtain the characteristic equation of $\left\langle X_{n}\right\rangle$ in the form $v^{2}-(2+i) v-$ $(1-i)=0$. Let $\gamma$ and $\delta$ be its two roots such that

$$
\gamma=\theta+1 \text { and } \delta=\vartheta+1
$$


Some noticeable points about $\gamma$ and $\delta$ are

$$
\gamma+\delta=2+i, \quad \gamma \delta=i-1=-(1-i) \text { and } \gamma-\delta=\sqrt{7}
$$

Lemma 3. For a square matrix $X=\left[\begin{array}{cc}2+i & 1-i \\ 1 & 0\end{array}\right]$ and $n \in \mathbb{Z}_{0}$, the following results hold

$$
\begin{aligned}
& {\left[\begin{array}{c}
X_{n+1} \\
X_{n}
\end{array}\right]=X^{n}\left[\begin{array}{c}
X_{1} \\
X_{0}
\end{array}\right]} \\
& X^{n}=(\gamma-\delta)^{-1}\left[\begin{array}{cc}
\gamma^{n+1}-\delta^{n+1} & -\delta \gamma^{n+1}+\gamma \delta^{n+1} \\
\gamma^{n}-\delta^{n} & -\delta \gamma^{n}+\gamma \delta^{n}
\end{array}\right]
\end{aligned}
$$

Proof. The equation (3.7) can be easily proved by the induction method. Certainly $X$ is a square matrix and let $v$ be the eigen value of $X$. Then by Cayley Hamilton theorem the characteristic equation of $X$ is given by the equation

$$
\begin{aligned}
|X-u I| & =0 \\
\left|\begin{array}{cc}
2+i & 1-i \\
1 & 0
\end{array}\right| & =0 \\
v^{2}-(2+i) v-(1-i) & =0
\end{aligned}
$$

Let $\gamma$ and $\delta$ be the characteristic roots as well as eigen values of matrix $X$. Now same as in [24] the eigen vectors corresponding to $\gamma$ and $\delta$ are $\left[\begin{array}{l}\gamma \\ 1\end{array}\right]$ and $\left[\begin{array}{l}\delta \\ 1\end{array}\right]$ respectively. Let $V_{1}=\left[\begin{array}{ll}\gamma & \delta \\ 1 & 1\end{array}\right]$ be the matrix of eigen vectors and $V_{2}=\left[\begin{array}{ll}\gamma & 0 \\ 0 & \delta\end{array}\right]$ is the diagonal matrix. Then by the process of diagonalization of matrices, we get

$$
\begin{aligned}
X^{n} & =V_{1} V_{2}^{n} V_{1}^{-1} \\
& =(\gamma-\delta)^{-1}\left[\begin{array}{ll}
\gamma & \delta \\
1 & 1
\end{array}\right]\left[\begin{array}{cc}
\gamma^{n} & 0 \\
0 & \delta^{n}
\end{array}\right]\left[\begin{array}{cc}
1 & -\delta \\
-1 & \gamma
\end{array}\right] \\
& =(\gamma-\delta)^{-1}\left[\begin{array}{cc}
\gamma^{n+1}-\delta^{n+1} & -\delta \gamma^{n+1}+\gamma \delta^{n+1} \\
\gamma^{n}-\delta^{n} & -\delta \gamma^{n}+\gamma \delta^{n}
\end{array}\right]
\end{aligned}
$$

Hence the proof of the equation (3.8). 
Theorem 4. (Binet's formula for the binomial form $\left\langle X_{n}\right\rangle$ ) For a square matrix $X=\left[\begin{array}{cc}2+i & 1-i \\ 1 & 0\end{array}\right]$ and $n \in \mathbb{Z}_{0}$, we have

$$
\begin{aligned}
X_{n} & =A \gamma^{n}+B \delta^{n}, \quad A=\frac{X_{1}-\delta X_{0}}{\gamma-\delta} \text { and } B=\frac{\gamma X_{0}-X_{1}}{\gamma-\delta} \\
& =s\left(\frac{\gamma^{n+1}-\delta^{n+1}}{\gamma-\delta}-\frac{\gamma^{n}-\delta^{n}}{\gamma-\delta}\right)-t\left(\gamma^{n}+\delta^{n}\right)
\end{aligned}
$$

Proof. Since $X=\left[\begin{array}{cc}2+i & 1-i \\ 1 & 0\end{array}\right]$ and $n \in \mathbb{Z}_{0}$ then clearly from the equation (3.8), we have

$$
X^{n}=(\gamma-\delta)^{-1}\left[\begin{array}{cc}
\gamma^{n+1}-\delta^{n+1} & -\delta \gamma^{n+1}+\gamma \delta^{n+1} \\
\gamma^{n}-\delta^{n} & -\delta \gamma^{n}+\gamma \delta^{n}
\end{array}\right]
$$

By using the equation (3.7), we have

$$
\begin{aligned}
{\left[\begin{array}{c}
X_{n+1} \\
X_{n}
\end{array}\right] } & =(\gamma-\delta)^{-1}\left[\begin{array}{cc}
\gamma^{n+1}-\delta^{n+1} & -\delta \gamma^{n+1}+\gamma \delta^{n+1} \\
\gamma^{n}-\delta^{n} & -\delta \gamma^{n}+\gamma \delta^{n}
\end{array}\right]\left[\begin{array}{l}
X_{1} \\
X 0
\end{array}\right] \\
& =(\gamma-\delta)^{-1}\left[\begin{array}{c}
X_{1} \gamma^{n+1}-X_{1} \delta^{n+1}-X_{0} \delta \gamma^{n+1}+X_{0} \gamma \delta^{n+1} \\
X_{1} \gamma^{n}-X_{1} \delta^{n}-X_{0} \delta \gamma^{n}+X_{0} \gamma \delta^{n}
\end{array}\right]
\end{aligned}
$$

Thus

$$
\begin{aligned}
X_{n} & =\frac{X_{1} \gamma^{n}-X_{1} \delta^{n}-X_{0} \delta \gamma^{n}+X_{0} \gamma \delta^{n}}{\gamma-\delta} \\
& =\frac{1}{\gamma-\delta}\left[\left(X_{1}-\delta X_{0}\right) \gamma^{n}+\left(\gamma X_{0}-X_{1}\right) \delta^{n}\right] \\
& =A \gamma^{n}+B \delta^{n}
\end{aligned}
$$

Where

$$
\begin{aligned}
A & =(\gamma-\delta)^{-1}\left(X_{1}-\delta X_{0}\right) \gamma^{n} \\
& =(\gamma-\delta)^{-1}[s(1+i)-t(2+i)-\delta(s-2 t)] \gamma^{n} \\
& =(\gamma-\delta)^{-1}(i s+s-i t-2 t-\delta s+2 \delta t) \gamma^{n} \\
& =(\gamma-\delta)^{-1}\left(i s \gamma^{n}+s \gamma^{n}-s \delta \gamma^{n}-i t \gamma^{n}-2 t \gamma^{n}+2 \delta t \gamma^{n}\right)
\end{aligned}
$$




$$
=(\gamma-\delta)^{-1}\left[i s \gamma^{n}+s \gamma^{n}-s(2+i-\gamma) \gamma^{n}-i t \gamma^{n}-2 t \gamma^{n}+2 t(2+i-\gamma) \gamma^{n}\right]
$$

By the Eqn. (3.6)

$$
\begin{aligned}
= & (\gamma-\delta)^{-1}\left(i s \gamma^{n}+s \gamma^{n}-2 s \gamma^{n}-i s \gamma^{n}+s \gamma^{n+1}-i t \gamma^{n}-2 t \gamma^{n}+4 t \gamma^{n}+i 2 t \gamma^{n}\right. \\
& \left.\quad-2 t \gamma^{n+1}\right) \\
= & (\gamma-\delta)^{-1}\left(-s \gamma^{n}+s \gamma^{n+1}+i t \gamma^{n}+2 t \gamma^{n}-2 t \gamma^{n+1}\right) \\
= & (\gamma-\delta)^{-1}\left[s \gamma^{n+1}-s \gamma^{n}+t \gamma^{n}(2+i-2 \gamma)\right]
\end{aligned}
$$

$$
\begin{aligned}
& =(\gamma-\delta)^{-1}\left[s \gamma^{n+1}-s \gamma^{n}+t \gamma^{n}(\gamma+\delta-2 \gamma)\right] \\
& =(\gamma-\delta)^{-1}\left[s \gamma^{n+1}-s \gamma^{n}-t \gamma^{n}(\gamma-\delta)\right]
\end{aligned}
$$

Similarly

$$
B=(\gamma-\delta)^{-1}\left[-s \delta^{n+1}+s \delta^{n}-t \delta^{n}(\gamma-\delta)\right]
$$

Therefore, we have

$$
\begin{aligned}
X_{n} & =\frac{1}{\gamma-\delta}\left[s \gamma^{n+1}-s \gamma^{n}-t \gamma^{n}(\gamma-\delta)-s \delta^{n+1}+s \delta^{n}-t \delta^{n}(\gamma-\delta)\right] \\
& =\frac{1}{\gamma-\delta}\left[s \gamma^{n+1}-s \delta^{n+1}-s \gamma^{n}+s \delta^{n}-t \gamma^{n}(\gamma-\delta)-t \delta^{n}(\gamma-\delta)\right] \\
& =s\left(\frac{\gamma^{n+1}-\delta^{n+1}}{\gamma-\delta}-\frac{\gamma^{n}-\delta^{n}}{\gamma-\delta}\right)-t\left(\gamma^{n}+\delta^{n}\right)
\end{aligned}
$$

Hence the result.

For the sake of convenience we express the large sized equation (3.12) into two sequences $\left\langle M_{n}\right\rangle$ and $\left\langle N_{n}\right\rangle$. Then, we have

$$
X_{n}=s\left(M_{n+1}-M_{n}\right)-t N_{n}, \quad n \in \mathbb{Z}_{0}
$$

Clearly

$$
M_{n}=\frac{\gamma^{n}-\delta^{n}}{\gamma-\delta} \text { and } N_{n}=\gamma^{n}+\delta^{n}, n \in \mathbb{Z}_{0}
$$

The following corollaries are useful while proving the subsequent results.

Corollary 5. (Binet's formula of the $(s, t)$-Jacobsthal sequence $\left\langle U_{n}\right\rangle$ ) Let $n \geq 0$, we have

$$
U_{n}=s \frac{\theta^{n+1}-\vartheta^{n+1}}{\theta-\vartheta}-t\left(\theta^{n}+\vartheta^{n}\right)
$$


Proof. From the equation (3.11), we have

$$
\begin{aligned}
X_{n} & =\frac{1}{\gamma-\delta}\left[s \gamma^{n+1}-s \delta^{n+1}-s \gamma^{n}+s \delta^{n}-t \gamma^{n}(\gamma-\delta)-t \delta^{n}(\gamma-\delta)\right] \\
& =s \frac{\gamma^{n}(\gamma-1)-\delta^{n}(\delta-1)}{\gamma-\delta}-t\left(\gamma^{n}+\delta^{n}\right) \\
& =s \frac{(\theta+1)^{n} \theta-(\vartheta+1)^{n} \vartheta}{\theta-\vartheta}-t\left[(\theta+1)^{n}+(\vartheta+1)^{n}\right] \quad \text { By the Eqn. } \\
& =s \frac{\sum_{l=0}^{n}\left(\begin{array}{l}
n \\
l
\end{array}\right) \theta^{l} \theta-\sum_{l=0}^{n}\left(\begin{array}{l}
n \\
l
\end{array}\right) \vartheta^{l} \vartheta}{\theta-\vartheta}-t\left[\sum_{l=0}^{n}\left(\begin{array}{l}
n \\
l
\end{array}\right) \theta^{l}+\sum_{l=0}^{n}\left(\begin{array}{l}
n \\
l
\end{array}\right) \vartheta^{l}\right] \\
& =\sum_{l=0}^{n}\left(\begin{array}{l}
n \\
l
\end{array}\right)\left[s \frac{\theta^{l+1}-\vartheta^{l+1}}{\theta-\vartheta}-t\left(\theta^{l}+\vartheta^{l}\right)\right]
\end{aligned}
$$

If we compare the equations (3.1) and (3.15), we get

$$
U_{l}=s \frac{\theta^{l+1}-\vartheta^{l+1}}{\theta-\vartheta}-t\left(\theta^{l}+\vartheta^{l}\right) \text { or } U_{n}=s \frac{\theta^{n+1}-\vartheta^{n+1}}{\theta-\vartheta}-t\left(\theta^{n}+\vartheta^{n}\right)
$$

Hence the result.

Corollary 6. For $m, n \geq 1$, the sequences $\left\langle X_{n}\right\rangle,\left\langle M_{n}\right\rangle$ and $\left\langle N_{n}\right\rangle$ satisfy the following properties

$$
\begin{aligned}
M_{m+n-1} & =\frac{X_{0} X_{m+n}-X_{1} X_{m+n-1}}{X_{0} X_{2}-X_{1}^{2}} \\
M_{m+n-1} & =\frac{N_{0} N_{m+n}-N_{1} N_{m+n-1}}{(\gamma-\delta)^{2}} \\
M_{m+n-1} & =\frac{M_{m} \gamma^{n}-M_{m} \delta^{n}-M_{m-1} \delta \gamma^{n}+M_{m-1} \gamma \delta^{n}}{\gamma-\delta} \\
N_{m+n} & =M_{m+n+1}+(1-i) M_{m+n-1}
\end{aligned}
$$

Proof. The proof of all the equations can be given by using the equations (3.9) and (3.14).

Corollary 7. If $m, q \in \mathbb{Z}_{0}$ and $n \in \mathbb{N}$, we get

$$
\begin{aligned}
N_{m} X_{m n+q}-(i-1)^{m} X_{m(n-1)+q} & =X_{m(n+1)+q} \\
N_{m} M_{m n+q}-(i-1)^{m} M_{m(n-1)+q} & =M_{m(n+1)+q}
\end{aligned}
$$

Proof. The proof is clearly seen by the equations (3.9) and (3.14). 


\section{Some GENERALIZED RESUlts}

In this section we obtain the various generalized results for the sequences $\left\langle X_{n}\right\rangle$ and $\left\langle M_{n}\right\rangle$ whose terms of the form $m n+q, m, n, q \geq 0$.

Theorem 8. (Generalized sum for $\left\langle X_{n}\right\rangle$ ) For $m, n, q \in \mathbb{Z}_{0}$, we have

$$
\sum_{j=1}^{n} X_{m j+q}=\frac{X_{m(n+1)+q}-(i-1)^{m} X_{m n+q}-X_{m+q}+(i-1)^{m} X_{q}}{N_{m}-(i-1)^{m}-1}
$$

Proof. Let

$$
S=\sum_{j=1}^{n} X_{m j+q}
$$

Multiplying both sides by $\left[N_{m}-(i-1)^{m}-1\right]$, we have

$$
S\left[N_{m}-(i-1)^{m}-1\right]=N_{m} \sum_{j=1}^{n} X_{m j+q}-(i-1)^{m} \sum_{j=1}^{n} X_{m j+q}-\sum_{j=1}^{n} X_{m j+q}
$$

Let

$$
S\left[N_{m}-(i-1)^{m}-1\right]=S_{1}+S_{2}+S_{3}
$$

Here

$$
S_{1}=N_{m} \sum_{j=1}^{n} X_{m j+q}
$$

Now

$$
\begin{aligned}
S_{2} & =-(i-1)^{m} \sum_{j=1}^{n} X_{m j+q} \\
& =-(i-1)^{m}\left(X_{m+q}+X_{2 m+q}+\cdots+X_{m n+q}\right)
\end{aligned}
$$

Add and subtract $X_{q}$ on R. H. S, we get

$$
\begin{aligned}
S_{2} & =-(i-1)^{m}\left(X_{m n+q}-X_{q}+X_{q}+X_{m+q}+X_{2 m+q}+\cdots+X_{m(n-1)+q}\right) \\
& =-(i-1)^{m} X_{m n+q}+(i-1)^{m} X_{q}-(i-1)^{m} \sum_{j=1}^{n} X_{m(j-1)+q}
\end{aligned}
$$


and

$$
S_{3}=-\sum_{j=1}^{n} X_{m j+q}
$$

Add and subtract $X_{m(n+1)+q}$ on R. H. S, we have

$$
\begin{aligned}
S_{3} & =-\left(-X_{m(n+1)+q}+X_{m+q}+X_{2 m+q}+\cdots+X_{m n+q}+X_{m(n+1)+q}\right) \\
& =X_{m(n+1)+q}-X_{m+q}-\sum_{j=1}^{n} X_{m(j+1)+q}
\end{aligned}
$$

Thus, we have

$$
\begin{aligned}
& S\left[N_{m}-(i-1)^{m}-1\right] \\
& =X_{m(n+1)+q}-(i-1)^{m} X_{m n+q}-X_{m+q}+(i-1)^{m} X_{q}+\sum_{j=1}^{n}\left(N_{m} X_{m j+q}\right. \\
& \left.\quad-(i-1)^{m} X_{m(j-1)+q}-X_{m(j+1)+q}\right) \\
& =X_{m(n+1)+q}-(i-1)^{m} X_{m n+q}-X_{m+q}+(i-1)^{m} X_{q}+\sum_{j=1}^{n}\left(X_{m(j+1)+q}\right. \\
& \quad-X_{m(j+1)+q} \quad \text { By the Eqn. }(3.20) \\
& =X_{m(n+1)+q}-(i-1)^{m} X_{m n+q}-X_{m+q}+(i-1)^{m} X_{q}
\end{aligned}
$$

Hence

$$
\sum_{j=1}^{n} X_{m j+q}=\frac{X_{m(n+1)+q}-(i-1)^{m} X_{m n+q}-X_{m+q}+(i-1)^{m} X_{q}}{N_{m}-(i-1)^{m}-1}
$$

This completes the proof of the theorem.

Theorem 9. For $m, n, q \in \mathbb{Z}_{0}$, the following property holds for $\left\langle M_{n}\right\rangle$

$$
\sum_{j=1}^{n} M_{m j+q}=\frac{M_{m(n+1)+q}-(i-1)^{m} M_{m n+q}-M_{m+q}+(i-1)^{m} M_{q}}{N_{m}-(i-1)^{m}-1}
$$

Theorem 10. If $x \in \mathbb{R}$ and $0 \leq m \leq q$, we get

$$
\sum_{n=0}^{\infty} M_{m n+q}=\frac{M_{q}+(i-1)^{-q} M_{m+q} x}{(i-1)^{m} x^{2}-N_{m} x+1}
$$


Proof. Let

$$
T=\sum_{n=0}^{\infty} M_{m n+q} x^{n}
$$

Multiplying both sides by $\left[(i-1)^{m} x^{2}-N_{m} x+1\right]$, we obtain

$$
\begin{aligned}
& T\left[(i-1)^{m} x^{2}-N_{m} x+1\right] \\
& =(i-1)^{m} \sum_{n=0}^{\infty} M_{m n+q} x^{n+2}-N_{m} \sum_{n=0}^{\infty} M_{m n+q} x^{n+1}+\sum_{n=0}^{\infty} M_{m n+q} x^{n} \\
& =(i-1)^{m} \sum_{n=0}^{\infty} M_{m n+q} x^{n+2}-N_{m} M_{q} x-N_{m} \sum_{n=0}^{\infty} M_{m(n+1)+q} x^{n+2}+M_{q}+M_{m+q} x \\
& \quad+\sum_{n=0}^{\infty} M_{m(n+2)+q} x^{n+2} \quad \text { By the Eqn. (3.21) } \\
& \left.=(i-1)^{m} M_{m n+q}\right] x^{n+2} \quad+\left(M_{m+q}-N_{m} M_{q}\right) x+\sum_{n=0}^{\infty}\left[M_{m(n+2)+q}-N_{m} M_{m(n+1)+q}\right. \\
& =M_{q}-(i-1)^{m} M_{q-m} x+\sum_{n=0}^{\infty}\left(M_{m(n+2)+q}-M_{m(n+2)+q} x^{n+2}\right.
\end{aligned}
$$

Since $-(i-1)^{m} M_{q-m}=(i-1)^{-q} M_{m+q}$, we get

$$
T\left[(i-1)^{m} x^{2}-N_{m} x+1\right]=M_{q}+(i-1)^{-q} M_{m+q} x
$$

Therefore, we have

$$
\sum_{n=0}^{\infty} M_{m n+q} x^{n}=\frac{M_{q}+(i-1)^{-q} M_{m+q} x}{(i-1)^{m} x^{2}-N_{m} x+1}
$$

Hence the proof.

\section{Matrix SeqUence of the Binomial Form $\left\langle X_{n}\right\rangle$}

In this section we define a kind type of matrix sequence $\left\langle Z_{n}\right\rangle$ to binomial form $\left\langle X_{n}\right\rangle$. In addition to this we investigate some results for the matrix sequence $\left\langle Z_{n}\right\rangle$. 
Definition 3. For $i(=\sqrt{-1})$, the matrix sequence $\left\langle Z_{n}\right\rangle_{n \in \mathbb{N}}$ is defined by the following equation

$$
Z_{n+1}=(2+i) Z_{n}+(1-i) Z_{n-1}, \quad n \geq 1
$$

with $Z_{0}=\left[\begin{array}{ll}M_{2} & (1-i) M_{1} \\ M_{1} & (1-i) M_{0}\end{array}\right]$ and $Z_{1}=\left[\begin{array}{cc}M_{3} & (1-i) M_{2} \\ M_{2} & (1-i) M_{1}\end{array}\right]$

Lemma 11. For a square matrix $X=\left[\begin{array}{cc}2+i & 1-i \\ 1 & 0\end{array}\right]$ and $n \geq 0$, we have

$$
\left[\begin{array}{c}
Z_{n+1} \\
Z_{n}
\end{array}\right]=X^{n}\left[\begin{array}{c}
Z_{1} \\
Z_{0}
\end{array}\right]
$$

Theorem 12. For a square matrix $X=\left[\begin{array}{cc}2+i & 1-i \\ 1 & 0\end{array}\right]$ and $n \geq 0$, the $n^{\text {th }}$ term of matrix sequence $\left\langle Z_{n}\right\rangle$ is given as

$$
\begin{aligned}
Z_{n} & =\left[\begin{array}{cc}
M_{n+2} & (1-i) M_{n+1} \\
M_{n+1} & (1-i) M_{n}
\end{array}\right] \\
& =\left(X_{0} X_{2}-X_{1}^{2}\right)^{-1}\left[\begin{array}{cc}
X_{0} X_{n+3}-X_{1} X_{n+2} & (1-i)\left(X_{0} X_{n+2}-X_{1} X_{n+1}\right) \\
X_{0} X_{n+2}-X_{1} X_{n+1} & (1-i)\left(X_{0} X_{n+1}-X_{1} X_{n}\right)
\end{array}\right] \\
& =(\gamma-\delta)^{-2}\left[\begin{array}{ll}
N_{0} N_{n+3}-N_{1} N_{n+2} & (1-i)\left(N_{0} N_{n+2}-N_{1} N_{n+1}\right) \\
N_{0} N_{n+2}-N_{1} N_{n+1} & (1-i)\left(N_{0} N_{n+1}-N_{1} N_{n}\right)
\end{array}\right]
\end{aligned}
$$

Proof. Since $X=\left[\begin{array}{cc}2+i & 1-i \\ 1 & 0\end{array}\right]$ then by the equation (3.8), we have

$$
X^{n}=(\gamma-\delta)^{-1}\left[\begin{array}{cc}
\gamma^{n+1}-\delta^{n+1} & -\delta \gamma^{n+1}+\gamma \delta^{n+1} \\
\gamma^{n}-\delta^{n} & -\delta \gamma^{n}+\gamma \delta^{n}
\end{array}\right]
$$

Since $\left[\begin{array}{c}Z_{n+1} \\ Z_{n}\end{array}\right]=X^{n}\left[\begin{array}{c}Z_{1} \\ Z_{0}\end{array}\right]$, we have

$$
\left[\begin{array}{c}
Z_{n+1} \\
Z_{n}
\end{array}\right]=(\gamma-\delta)^{-1}\left[\begin{array}{cc}
\gamma^{n+1}-\delta^{n+1} & -\delta \gamma^{n+1}+\gamma \delta^{n+1} \\
\gamma^{n}-\delta^{n} & -\delta \gamma^{n}+\gamma \delta^{n}
\end{array}\right]\left[\begin{array}{c}
Z_{1} \\
Z_{0}
\end{array}\right]
$$


A. A. Wani, S. Halici, T. A. Tarray - On a Study of Binomial Form ...

$$
=(\gamma-\delta)^{-1}\left[\begin{array}{c}
Z_{1} \gamma^{n+1}-Z_{1} \delta^{n+1}-Z_{0} \delta \gamma^{n+1}+Z_{0} \gamma \delta^{n+1} \\
Z_{1} \gamma^{n}-Z_{1} \delta^{n}-Z_{0} \delta \gamma^{n}+Z_{0} \gamma \delta^{n}
\end{array}\right]
$$

Therefore

$$
\begin{aligned}
Z_{n}= & \frac{Z_{1} \gamma^{n}-Z_{1} \delta^{n}-Z_{0} \delta \gamma^{n}+Z_{0} \gamma \delta^{n}}{\gamma-\delta} \\
= & \frac{1}{\gamma-\delta}\left\{\left[\begin{array}{rr}
M_{3} & (1-i) M_{2} \\
M_{2} & (1-i) M_{1}
\end{array}\right] \gamma^{n}-\left[\begin{array}{rr}
M_{3} & (1-i) M_{2} \\
M_{2} & (1-i) M_{1}
\end{array}\right] \delta^{n}-\left[\begin{array}{cc}
M_{2} & (1-i) M_{1} \\
M_{1} & (1-i) M_{0}
\end{array}\right] \delta \gamma^{n}\right. \\
& \left.+\left[\begin{array}{ll}
M_{2} & (1-i) M_{1} \\
M_{1} & (1-i) M_{0}
\end{array}\right] \gamma \delta^{n}\right\}
\end{aligned}
$$

Hence the result.

Corollary 13. If $n \geq 0$, the following relation is true for the sequences $\left\langle X_{n}\right\rangle,\left\langle M_{n}\right\rangle$ and $\left\langle N_{n}\right\rangle$

$$
N_{n+1}=\frac{X_{0}\left[X_{n+3}+(1-i) X_{n+1}\right]-X_{1}\left[X_{n+2}+(1-i) X_{n}\right]}{X_{0} X_{2}-X_{1}^{2}}
$$

Proof. If we compare corresponding terms of matrices from the equations (5.3) and (5.4). Therefore we have

$$
M_{n+2}=\frac{X_{0} X_{n+3}-X_{1} X_{n+2}}{X_{0} X_{2}-X_{1}^{2}}
$$




$$
(1-i) M_{n}=(1-i) \frac{X_{0} X_{n+1}-X_{1} X_{n}}{X_{0} X_{2}-X_{1}^{2}}
$$

If we add these equations together, we have

$$
\begin{aligned}
M_{n+2}+(1-i) M_{n} & =\frac{X_{0}\left[X_{n+3}+(1-i) X_{n+1}\right]-X_{1}\left[X_{n+2}+(1-i) X_{n}\right]}{X_{0} X_{2}-X_{1}^{2}} \\
N_{n+1} & =\frac{X_{0}\left[X_{n+3}+(1-i) X_{n+1}\right]-X_{1}\left[X_{n+2}+(1-i) X_{n}\right]}{X_{0} X_{2}-X_{1}^{2}}
\end{aligned}
$$

By the Eqn. (3.19)

This proves the equation (5.6).

Theorem 14. (Sum of the first $n$ terms of matrix sequence $\left\langle Z_{n}\right\rangle$ )

$\sum_{j=1}^{n} Z_{n}=2^{-1}\left[\begin{array}{cc}M_{n+3}-(1-i) M_{n+2}-(7+2 i) & (1-i) M_{n+2}-2 i M_{n+1}-3(1-i) \\ M_{n+2}-(1-i) M_{n+1}-3 & (1-i) M_{n+1}-2 i M_{n}-(1-i)\end{array}\right]$

Proof. From the equations (4.2) and (5.3), we have

$$
\begin{aligned}
\sum_{j=1}^{n} Z_{n} & =\sum_{j=1}^{n}\left[\begin{array}{cc}
M_{n+2} & (1-i) M_{n+1} \\
M_{n+1} & (1-i) M_{n}
\end{array}\right] \\
& =\left[\begin{array}{cc}
\frac{M_{n+3}+(1-i) M_{n+2}-(7+2 i)}{2} & (1-i) \frac{M_{n+2}+(1-i) M_{n+1}-3}{2} \\
\frac{M_{n+2}+(1-i) M_{n+1}-3}{2} & (1-i) \frac{M_{n+1}+(1-i) M_{n}-1}{2}
\end{array}\right] \\
& =2^{-1}\left[\begin{array}{cc}
M_{n+3}-(1-i) M_{n+2}-(7+2 i) & (1-i) M_{n+2}-2 i M_{n+1}-3(1-i) \\
M_{n+2}-(1-i) M_{n+1}-3 & (1-i) M_{n+1}-2 i M_{n}-(1-i)
\end{array}\right]
\end{aligned}
$$

Hence the proof.

Theorem 15. (Generating function of the binomial matrix sequence $\left\langle Z_{n}\right\rangle$ ) Let $x \in \mathbb{R}$, we get

$$
\sum_{j=1}^{n} Z_{n} x^{n}=\left[\begin{array}{cc}
\frac{2+i+(i-1)^{-2}(4+3 i) x}{(i-1) x^{2}-(2+i) x+1} & \frac{1-i-(2+i) x}{(i-1) x^{2}-(2+i) x+1} \\
\frac{1+(i-1)^{-1}(2+i) x}{(i-1) x^{2}-(2+i) x+1} & \frac{(1-i)}{(i-1) x^{2}-(2+i) x+1}
\end{array}\right]
$$


A. A. Wani, S. Halici, T. A. Tarray - On a Study of Binomial Form ...

Proof. The proof of this theorem is established by using the equations (5.3) and (4.3).

\section{REFERENCES}

[1] Koshy T. Fibonacci and Lucas numbers with Applications. John Wiley and Sons, 2011.

[2] A. F. Horadam, A Generalized Fibonacci Sequence, The American Mathematical Monthly, 68(5) (1961), 455-459.

[3] A. F. Horadam, Basic Properties of a Certain Generalized Sequence of Numbers, The Fibonacci Quarterly, 3(3) (1965), 161-176.

[4] A. F. Horadam, Complex Fibonacci Numbers and Fibonacci Quaternions. The American Mathematical Monthly, 70(3) (1963), 289-291.

[5] J. H. Jordan, Gaussian Fibonacci and Lucas Numbers, Fibonacci Quarterly, 3(4) (1965), 315-318.

[6] G. Berzsenyi, Gaussian Fibonacci Numbers, Fibonacci Quarterly, 15(3) (1977), 233-236.

[7] C. J. Harman, Complex Fibonacci Numbers, Fibonacci Quarterly, 19(1), (1981), $82-86$.

[8] S. Pethe and A. F. Horadam, Generalised Gaussian Fibonacci Numbers, Bulletin of the Australian Mathematical Society, 33(1) (1986), 37-48.

[9] M. Asci and E. Gurel, Gaussian Jacobsthal Lucas Numbers, Ars Combinatoria , 111 (2013), 53-63.

[10] M. Asci and E. Gurel, Gaussian Jacobsthal and Gaussian Jacobsthal Lucas Polynomials, Notes on Number Theory and Discrete Mathematics, 19(1) (2013), 2536.

[11] P. Catarino, P. Vasco, H. Campos, A. P. Aires, and A. Borges, New families of Jacobsthal and Jacobsthal-Lucas Numbers, Algebra and Discrete Mathematics, 20(1), (2015), 40-54.

[12] S. Uygun, A New Generalization for Jacobsthal and Jacobsthal Lucas Sequences, Asian Journal of Mathematics and Physics, 2(1) (2018), 14-21.

[13] K. W. Chen, Identities from the Binomial Transform, Journal of Number Theory, 124(1) (2007), 142-150.

[14] K. Wójcik, Binomial Transform and Dold Sequences, Journal of Integer Sequences, 18(2) (2015), 1-18.

[15] A. A. Wani, S. A. Bhat and G. P. S. Rathore, Fibonacci-Like Sequence Associated with k-Pell, k-Pell-Lucas and Modified k-Pell Sequences, 16(2) (2017), 159-171. 
A. A. Wani, S. Halici, T. A. Tarray - On a Study of Binomial Form ...

[16] H. Civic, and R. Turkmen, On the $(s, t)$-Fibonacci and Matrix Sequences, Ars Combinatoria , 87 (2008), 161-173.

[17] Y. Yazlik, N. Taskara, K. Uslu, and N. Yilmaz, The Generalized- $(s, t)$ Sequence and its matrix Sequence, AIP Conference Proceedings(International Conference on Numerical Analysis and Applied Mathematics, Greece 19-25 September 2011), 1389(1) (2011), 381-384, http://doi.org/10.1063/1.3636742.

[18] A. Ipek, K. Ari, and R. Turkmen, The Generalized- $(s, t)$ Fibonacci and Fibonacci Matrix Sequences, Transylvanian Journal of Mathematics and Mechanics, 7(2) (2015), 137-148.

[19] Y. Yazlik, N. Yilmaz, and N. Taskara, The Generalized-(s,t) Matrix Sequence's Binomial Transforms, General Mathematics Notes, 24(1) (2014), 127-136.

[20] Uygun S. The $(s, t)$-Jacobsthal and $(s, t)$-Jacobsthal Lucas Sequences. Applied Mathematical Sciences, 9(70), (2015), 3467-3476.

[21] K. Uslu, and S. Uygun, The $(s, t)$-Jacobsthal and $(s, t)$-Jacobsthal-Lucas Matrix Sequences, Ars Combinitoria, 108 (2013), 13-22.

[22] S. Uygun, Some Sum Formulas of $(s, t)$-Jacobsthal and $(s, t)$-Jacobsthal-Lucas Matrix Sequences, Applied Mathematics, 7(1) (2013), 61-69.

[23] S. Uygun, and K. Uslu, The $(s, t)$-Generalized Jacobsthal Matrix Sequences, Computational Analysis(Springer Proceedings in Mathematics and Statistics, Springer Cham) 155(23) (2016), 325-336, http://doi.org/10.1007/978-3-319-28443-9_23.

[24] A. A. Wani, G. P. S. Rathore, V. H. Badshah and K. Sisodiya, A Two-by-Two Matrix Representation of a Generalized Fibonacci Sequence, Hacettepe Journal of Mathematics and Statistics, 47(3) (2018), 637-648.

A. A. Wani

School of Studies in Mathematics,

Vikram University Ujjain,

Ujjain, India.

email: arfatahmadwani@gmail.com

S. Halici

Department of Mathematics, Faculty of Arts and Sciences,

Sakarya University,

54187 Sakarya, Iran.

email:shalici@pau.edu.tr

Tanveer Ahmad Tarray

Department of Mathematical Sciences, Islamic University of Science and Technology, 
A. A. Wani, S. Halici, T. A. Tarray - On a Study of Binomial Form ...

Awantipora,Jammu and Kashmir, India. email: tanveerstat@gmail.com 